\title{
PROBLEMS AND SOLUTIONS BY THE APPLICATION OF JULIA SET THEORY TO ONE-DOT AND MULTI-DOTS NUMERICAL METHODS
}

\author{
ANNA TOMOVA
}

(Received 6 February 2001)

\begin{abstract}
In 1977 Hubbard developed the ideas of Cayley (1879) and solved in particular the Newton-Fourier imaginary problem. We solve the Newton-Fourier and the ChebyshevFourier imaginary problems completely. It is known that the application of Julia set theory is possible to the one-dot numerical method like the Newton's method for computing solution of the nonlinear equations. The secants method is the two-dots numerical method and the application of Julia set theory to it is not demonstrated. Previously we have defined two one-dot combinations: the Newton's-secants and the Chebyshev's-secants methods and have used the escape time algorithm to analyse the application of Julia set theory to these two combinations in some special cases. We consider and solve the Newton's-secants and Tchebicheff's-secants imaginary problems completely.
\end{abstract}

2000 Mathematics Subject Classification. 37Fxx, 37F50.

1. Introduction. In 1879, Cayley [2] demonstrated the Newton-Fourier imaginary problem for $F(z)=z^{2}-C$. In 1977, Hubbard [3] solved this problem. Using the transformation $G(z)=\left(z+C^{1 / 2}\right) /\left(z-C^{1 / 2}\right)=u, G^{-1}(u)=C^{1 / 2}(u+1) /(u-1)$ he proved $[2,3]$ that the dynamical system

$$
C: f_{N}(z)=z-\frac{F(z)}{F^{\prime}(z)}
$$

and $C: R(u)=u^{2}$ are equivalent, that is, $R(u)=G \circ f_{N} \circ G^{-1}(u)=u^{2}$.

In [5] we define the Newton's-secants method for computing solutions of the nonlinear equation $F(z)=0$ : it tells us to consider the dynamical system, associated with $F(z)$

$$
C: f_{\mathrm{Ns}}(z)=f_{N}(z)-\frac{F\left(f_{N}(z)\right)\left(f_{N}(z)-z\right)}{F\left(f_{N}(z)\right)-F(z)},
$$

where $f_{N}(z)$ is the Newton transformation, associated with the function $F(z)$ (1.1).

In [6] we define the Chebyshev's secants method for computing the solution of the nonlinear equations: it tell us to consider the dynamical system

$$
C: f_{\mathrm{Chs}}(z)=f_{\mathrm{Ch}}(z)-\frac{F\left(f_{\mathrm{Ch}}(z)\right)\left(f_{\mathrm{Ch}}(z)-z\right)}{F\left(f_{\mathrm{Ch}}(z)\right)-F(z)},
$$

where

$$
f_{\mathrm{Ch}}(z)=z-\frac{F(z)}{F^{\prime}(z)}-\frac{F^{2}(z) F^{\prime \prime}(z)}{2\left(F^{\prime}(z)\right)^{3}}
$$


2. Main results. Consider the polynomial

$$
F(z)=z^{n}-C, \quad n \in N
$$

and the transformations

$$
G(z)=\frac{z-C^{1 / n}}{z+C^{1 / n}}, \quad G^{-1}(u)=C^{1 / n} \frac{u+1}{1-u} .
$$

We can prove the following theorems.

THEOREM 2.1. The dynamical system $f_{N}(z)(1.1)$ where $F(z)$ is (2.1) and

$$
R_{N}(u)=G \circ f_{N} \circ G^{-1}(u)=\frac{2 n u(u+1)^{n-1}-(u+1)^{n}+(1-u)^{n}}{2 n(u+1)^{n-1}-(u+1)^{n}+(1-u)^{n}}
$$

are locally equivalent in a sufficiently little circle around each of the $n$-roots of the polynomial $F(z)=z^{n}-C$. Since $C$ is generally a complex number, the value $C^{1 / n}$ is fixed as any of the $n$-different values of the $n$-roots of the polynomial (2.1). The fixed points of $R_{n}(u)$ are $u=1$ and $u=-i \tan (k p i / n), k=1,2, \ldots, n-1$.

THEOREM 2.2. The dynamical system (1.4) where $F(z)$ is (2.1) and

$$
\begin{aligned}
& R_{\mathrm{Ch}}(u) \\
& \quad=\frac{\left(-u^{2}+1\right)^{n}(4 n-2)+(u-1)^{2 n}(1-n)+(u+1)^{2 n-1}\left(4 n^{2} u-3 n(u+1)+u+1\right)}{\left(-u^{2}+1\right)^{n}(4 n-2)+(u-1)^{2 n}(1-n)+(u+1)^{2 n-1}\left(4 n^{2}-3 n(u+1)+u+1\right)}
\end{aligned}
$$

are locally equivalent in a sufficiently little open circle around each of the $n$-roots of the polynomial (2.1). The fixed points of (2.1) are

$$
\begin{gathered}
1, \quad-i \tan \frac{k p i}{n}, \quad \frac{e^{i 2 p i m / n}(n-1)^{1 / n} /(3 n-1)^{1 / n}-1}{e^{i 2 p i m / n}(n-1)^{1 / n} /(3 n-1)^{1 / n}+1}, \\
k=1,2, \ldots, n-1, m=0,1,2, \ldots, n-1 .
\end{gathered}
$$

We can prove two similar theorems about $f_{\mathrm{Ns}}(z)$ and $f_{\mathrm{Chs}}(z)$ in the case (2.1), but the general formulas for $R_{\mathrm{Ns}}(u)$ and $R_{\mathrm{Ch}}(u)$ are too long and we will publish them later. Here we will consider only some examples. Consider now the polynomial

$$
F(z)=\left(z^{n}-C\right)^{l}, \quad l, n \in N .
$$

We are familiar, from calculus, with the modifications of Newton's and Chebyshev's methods for computing the solution of the equation $F(z)=0$. They tell us to consider the dynamical systems

$$
\begin{aligned}
& C: f_{\mathrm{Nm}}(z)=z-\frac{u(z)}{u^{\prime}(z)}, \\
& C: f_{\mathrm{Chm}}(z)=z-\frac{u(z)}{u^{\prime}(z)}-\frac{u^{2}(z) u^{\prime \prime}(z)}{2 u^{\prime}(z)^{3}},
\end{aligned}
$$

where $u(z)=F(z) / F^{\prime}(z)$. We can prove two similar theorems about $f_{\mathrm{Nm}}(z)$ and $f_{\mathrm{Chm}}(z)$ in the case (2.6). We obtain that $R_{\mathrm{Nm}}(z)=R_{N}(z)$, but the general formula for $R_{\mathrm{Chm}}(z)$ is too long and we will publish it later. We will consider here only some examples. 
3. Examples and the application of Julia set theory. (1) Case $n=2$.

(a) Newton's-secants method, then

$$
f_{\mathrm{Ns}}(z)=\frac{z^{3}+3 C z}{3 z^{2}+C}
$$

The fixed points of $f_{\mathrm{Ns}}(z)$ are $0, C^{1 / 2}$, and $-C^{1 / 2}$. The first is repulsive: $f_{\mathrm{Ns}}^{\prime}(0)=3$ and the other are attractive: $f_{\mathrm{Ns}}^{\prime}\left(C^{1 / 2}\right)=f_{\mathrm{Ns}}^{\prime}\left(-C^{1 / 2}\right)=f_{\mathrm{Ns}}^{\prime \prime}\left(C^{1 / 2}\right)=f_{\mathrm{Ns}}^{\prime \prime}\left(-C^{1 / 2}\right)=0$, but $f_{\mathrm{Ns}}^{\prime \prime \prime}\left(C^{1 / 2}\right)=f_{\mathrm{Ns}}^{\prime \prime \prime}\left(-C^{1 / 2}\right)=3 / 2 C$. This is clear, because $R_{\mathrm{Ns}}(u)=u^{3}$. The Julia set for $u^{3}$ is $|u|=1$. This is the second case in the fractal geometry that the formula for Julia set is the same and too simple. (See [1, 2, 3].) It is clear too that the order of successive approximations is 3 [4], it is more than the order of Newton's approximations which is 2 and the order of secants' approximations which is $1,61803 \ldots$.. [4]. Consider the problem about the computation's efficiency in this case [3]. Assume that for the computation of $F(z), F^{\prime}(z)$, and $F\left(f_{n}(z)\right)$ are necessary 3 computation's units. Then the efficiency of the Newton's-secants method will be $3^{1 / 3} 1,442 \ldots$ that is between the efficiency of the secants methods $(1,61803 \ldots)$ and the sufficiency of the Newton's method $\left(2^{1 / 2} 1,414 \ldots\right)$. Assume that 4 computation's units are used, then the efficiency of the Newton-secants method will be $3^{1 / 4}$ that is more than the efficiency of the chords method which is one.

(b) Chebyshev's-secants method gives

$$
R_{\mathrm{Chs}}(u)=\frac{u^{4}(2+u)}{1+2 u}
$$

The fixed points of $R_{\mathrm{Chs}}(u)$ are 0,1 , and -1 . The first is attractive and in a little circle of $0, R_{\mathrm{Chs}}(u)$, and $R(u)=u^{3}$ are equivalent. The order of successive approximations is 4 , this is more than the Chebyshev's approximations order which is 3 [4]. The point -1 is parabolic and the point 1 is repulsive. The fixed point's type is determined by the facts that $R_{\text {Chs }}^{\prime}(0)=R_{\text {Chs }}^{\prime \prime}(0)=R_{\text {Chs }}^{\prime \prime \prime}(0)=0, R_{\text {Chs }}^{(4)}(0)=48, R_{\text {Chs }}(1)=11 / 3, R_{\text {Chs }}^{\prime}(-1)=1$. On the plate 1 , the escape time algorithm [1] is used to analyse the trajectories of $R_{\mathrm{Chs}}(u)$.

(c) Chebyshev's-modification method gives

$$
R_{\mathrm{Chm}}(z)=\frac{u^{4}\left(u^{2}+3\right)}{3 u^{2}+1}
$$

(2) Case $n=3$.

(a) Newton's method gives

$$
R_{N}(u)=\frac{2 u^{2}(u+3)}{3+3 u+3 u^{2}-u^{3}}
$$

On the plate 2, the escape time algorithm [1] is used to analyse the trajectories of $R_{N}(u)$.

(b) Chebyshev's method gives

$$
R_{\mathrm{Ch}}(u)=\frac{4 u^{3}\left(u^{3}+9 u^{2}+15 u+15\right)}{9+36 u+45 u^{2}+60 u^{3}+15 u^{4}-5 u^{6}}
$$


(c) Newton's-secants method gives

$$
R_{\mathrm{Ns}}(u)=\frac{4 u^{3}\left(u^{4}+18 u^{3}+60 u^{2}+54 u+27\right)}{27+81 u+171 u^{2}+189 u^{3}+177 u^{4}+27 u^{5}-23 u^{6}-9 u^{7}} .
$$

On the plates 3, 4 the escape time algorithm [1] is used to analyse the trajectories of $f_{\mathrm{Ns}}(z)$ in the case $F(z)=z^{3}-1$.
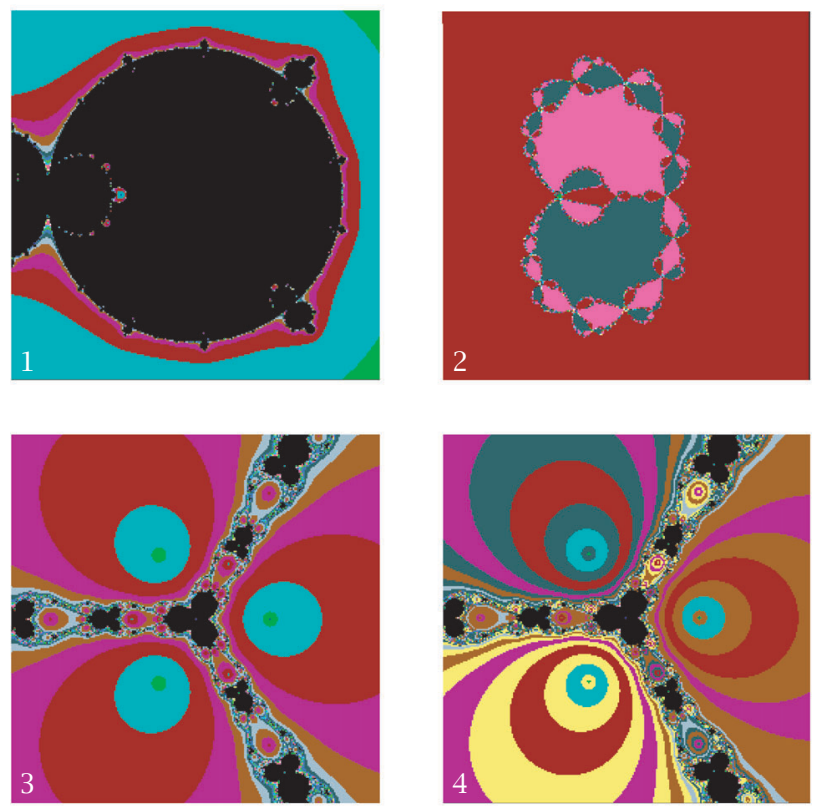

FiguRE 3.1. Plates 1-4.

\section{REFERENCES}

[1] M. Barnsley, Fractals Everywhere, Academic Press, Massachusetts, 1988. MR 90e:58080. Zbl 0691.58001.

[2] A. Cayley, The Newton-Fourier imaginary problem, Amer. J. Math. 2 (1879), 97.

[3] H.-O. Peitgen and P. H. Richter, The Beauty of Fractals. Images of Complex Dynamical Systems, Springer-Verlag, Berlin, 1986. MR 88e:00019. Zbl 0601.58003.

[4] B. Sendoff and V. Popoff, The Calculus Methods, Academic Press, Sofia University, Kliment, Sofia, 1996 (Bulgarian).

[5] A. Tomova, The Application of Julia set theory to Newton's-secants method, 25th Jubilee Summer School "Applications of Mathematics in Engineering and Economics" (Sozopol, Bulgaria), 1999.

[6] - The solution of the Chebyshev-Fourier imaginary problem for $F(z)=z^{n}-C, n \geq 2$, 25th Jubilee Summer School "Applications of Mathematics in Engineering and Economics” (Sozopol, Bulgaria), 2001, to appear.

AnNa Tomova: Department of Mathematics, Physics AND Informatics, Naval ACADEMY, VARNA, BULGARIA

E-mail address: anavioleta@mobi kom.com 


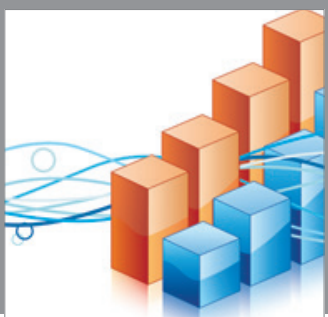

Advances in

Operations Research

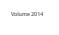

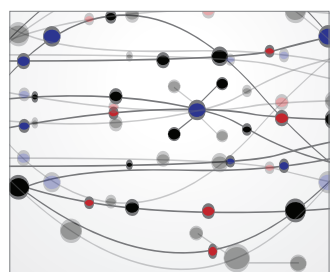

\section{The Scientific} World Journal
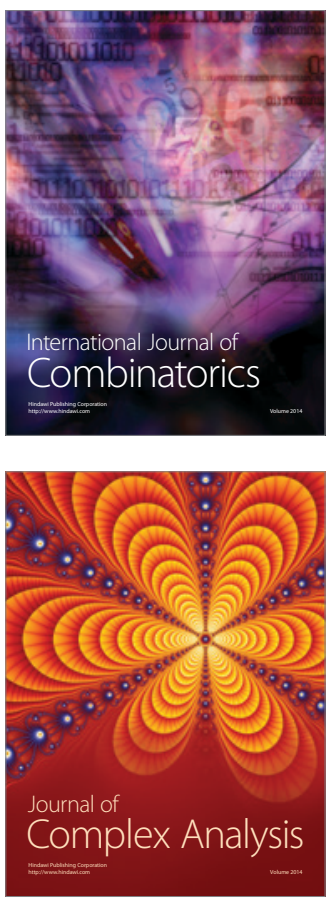

International Journal of

Mathematics and

Mathematical

Sciences
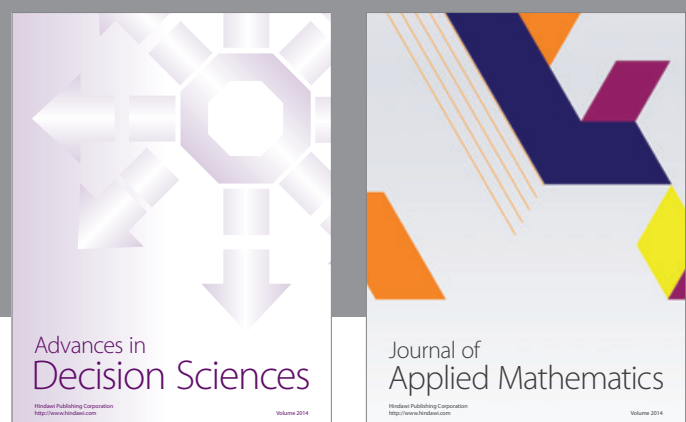

Journal of

Applied Mathematics
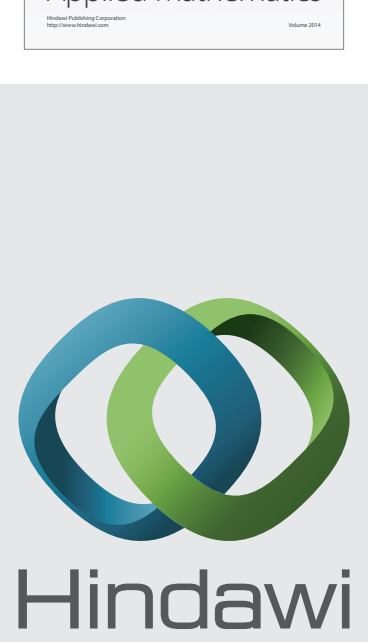

Submit your manuscripts at http://www.hindawi.com
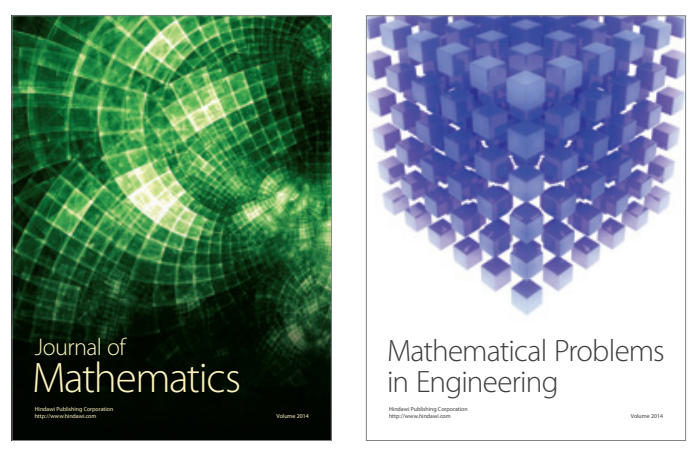

Mathematical Problems in Engineering
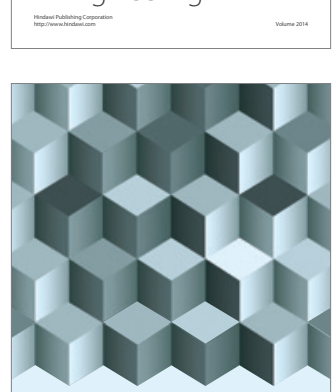

Journal of

Function Spaces
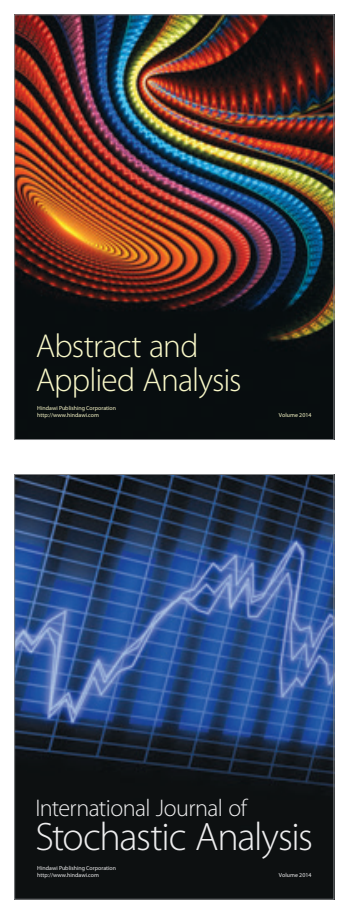

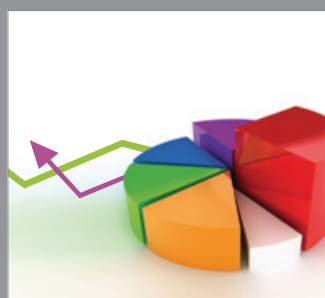

ournal of

Probability and Statistics

Promensencen
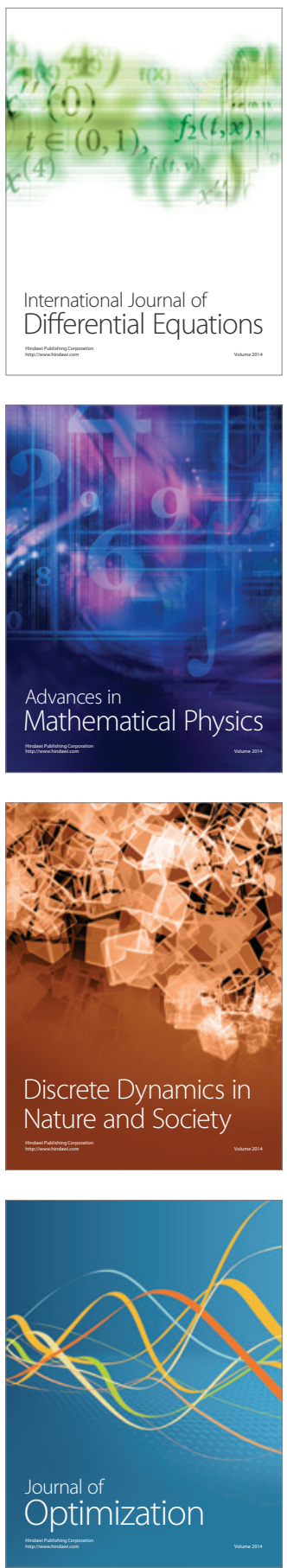